\title{
H. Eugene Stanley Selected for 1998 David Turnbull Lectureship for Contributions to Phase Transitions and Critical Phenomena
}

As an educator and scientific pioneer whose work over the past 35 years has opened subfields of materials research, H. Eugene Stanley of Boston University has been selected to deliver the 1998 David Turnbull Lecture. He is cited for "his insights into the statistical aspects of materials phenomena including phase transitions, pattern formation, and disordered, granular, and soft materials, and for his outstanding lecturing and writing on these topics."

The David Turnbull Lectureship recognizes the career of a scientist who has made outstanding contributions to understanding materials phenomena and properties through research, writing, and lecturing, as exemplified by David Turnbull.

The classic book, Introduction to Phase Transitions and Critical Phenomena (Oxford University Press), which Stanley wrote from 1968 to 1970 , has educated a generation of physicists worldwide. It won the 1971 Choice award for the best academic book of that year and has been translated into many languages, including Russian and Japanese. His other key publications in materials research include Biomedical Physics and Biomaterials Science (MIT Press), Disordered Materials and Interfaces (Materials Research Society), and Fractal Concepts in Surface Growth (Cambridge University Press) which is said to provide an overview of some of the most important developments in application of dynamic scaling concepts to surface growth.

In his studies of magnetism, started during his graduate studies, Stanley, with T.A. Kaplan at Harvard University, proposed a novel phase transition in the twodimensional $X Y$ and Heisenberg models as having a critical point without the appearance of long-range order, which was a revolutionary concept in the late 1960s. The 1967 Stanley-Kaplan phase transition stimulated the development of the subfield of low-dimensional materials. Following this work is Stanley's invention, in 1968, of the $n$-vector model of magnetism, which in turn led to major advances in fundamental understanding

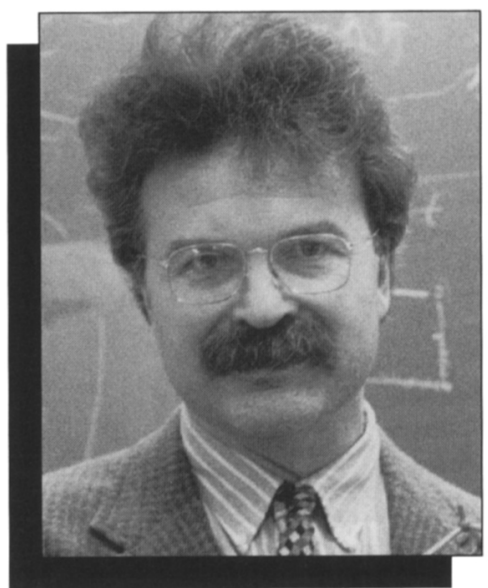

H. Eugene Stanley

of polymer materials since it became possible to treat polymers by taking Stanley's model to the $n \rightarrow 0$ limit.

Setting Stanley apart from many theorists is his collaboration with expert experimentalists, as is exemplified in his work on glasslike phases of metastable water. In a series of papers starting in 1992, Stanley and his students have proposed the twocritical-point hypothesis, which helped initiate a new field of research known as liquid polyamorphism. The experimentally observed phase transition between lowdensity amorphous (LDA) ice and highdensity amorphous (HDA) ice is the structurally arrested manifestation of an underlying equilibrium liquid-liquid transition between two distinct forms of liquid water. In the March 12, 1998 issue of Nature, Stanley and O. Mishima in Japan published experiments probing the new lowtemperature domain of the phase diagram of water. Stanley's work in this area, accomplished over nearly two decades, has far-reaching implications in various fields, including materials science and the technology of low-temperature preservation of biological molecules.

In the area of disordered materials, Stanley and his students have recently contributed the "directed percolation depinning" model to describe surface growth in the presence of frozen-in disor- der. This model explains how the wet front moves forward by a series of avalanches as it invades the disordered medium. Another topic on which Stanley worked is on the theoretical modeling of nanostructure growth which incorporates deposition, particle, and cluster diffusion. Modeling has allowed the understanding in many experiments of thin-film deposition. Stanley and his students have also reported in Nature (1997) the ordering phenomenon of spontaneous self-stratification into alternating layers when a mixture of grains of two different sizes is poured into a heap. This latter phenomenon was named as the "top physics discovery of $1997^{\prime \prime}$ in the January 1998 issue of Discover.

A few years after obtaining his PhD degree in physics from Harvard (1967), Stanley achieved the Herman von Helmholtz Associate Professorship in 1973 at the Massachusetts Institute of Technology in recognition of his interdepartmental teaching and research with the Harvard-MIT Program in Health Sciences and Technology. He joined Boston University in 1976. Stanley has mentored numerous students ranging from school children to $\sim 60 \mathrm{PhD}$ candidates and $\sim 60$ postdoctoral research associates, many of whom now hold leadership positions in the materials community.

He has co-founded a series of NATO Advanced Study Institutes in interdisciplinary physics, co-directed the 1996 Enrico Fermi School of Physics on Complex Systems, and has pressed for the reform of medical education through the introduction of concepts and techniques of the physical sciences. He has presented over 50 opening, keynote, plenary, or closing addresses and 200 principal invited talks, published over 500 journal articles, and has received many honors, including Honorary Professor at Eotvos Lorand University in Budapest and Doctorates Honoris Causa from Bar-Ilan University and the Eotvos Lorand University.

Stanley will present his lecture, "Unsolved Mysteries of Water in Its Liquid and Glass Phases," on December 2,1998 , at $12: 15$ p.m. in Salon $E$ at the Boston Marriott.

\section{The Materials Research Society publishes a series of symposium proceedings as well as materials research related books. An online catalog is available at http://www.mrs.org/publications/}

